\title{
Gravidez recorrente na adolescência e vulnerabilidade social no Rio de Janeiro (RJ, Brasil): uma análise de dados do Sistema de Nascidos Vivos
}

Katia Silveira da Silva ${ }^{1}$

Riva Rozenberg ${ }^{2}$

Claudia Bonan ${ }^{1}$

Vânia Cristina Costa Chuva ${ }^{1}$

Simoni Furtado da Costa ${ }^{1}$

Maria Auxiliadora de Souza Mendes Gomes ${ }^{1}$

${ }^{1}$ Instituto Fernandes Figueira, Fundação Oswaldo Cruz. Av. Rui Barbosa 716, Flamengo. 22500-050 Rio de Janeiro RJ.

katiass@iff.fiocruz.br

${ }^{2}$ Secretaria Estadual de Saúde e Defesa Civil do Rio de Janeiro.
Abstract Repeated pregnancy (RP) among adolescents is seldom researched in Brazil, even tough the debate on the reproductive rights is important for this extract of population. A transversal study was developed with data from the Declaration of Live Births of adolescent mothers, living in Rio de Janeiro (RJ, Brazil), in 2005. The aim was to estimate the magnitude and features associated with $R P$. Prevalence ratios $(P R)$ of $R P$, with 95\% confidence interval (CI) for selected variables, were estimated through log-binomial multivariate regression. Among 12,168 adolescents, a $R P$ prevalence of $29.1 \%$ was identified and the principal factors associated were: age 15-19 anos $(P R=5.42 ; R I$ 95\% 3.72-7.81); not doing prenatal consultation ( $R P=2.36$; $C I$ 95\% 2.16-2.58); educational status $<4$ anos $(P R=1.48$; CI 95\% 1.25 1.76); housewife job ( $P R=1.8$; CI 95\% 1.57-2.15) or other $(P R=1.9 ; C I 95 \% ; 1.73-2.10)$. Giving birth by cesarean section and low birth weight were negatively associated to repeated pregnancy with $R P$ equal to 0,94 (CI 95\%; 0,86-0,99) and 0.69 (CI 95\%; 0.62-0.77). The adolescents with RP had worst socioeconomic and assistance indicators than those on their first pregnancy. Specific social policies for adolescent mothers, in vulnerable situation, will enable them to have better conditions to exercise their reproductive rights.

Key words Adolescent pregnancy, Social vulnerability, Multiparity
Resumo A recorrência da gravidez na adolescência é tema pouco estudado no Brasil, sendo necessário o debate dos direitos reprodutivos dessa parcela da população. Realizou-se estudo transversal com dados das Declarações de Nascidos Vivos de mães adolescentes residentes na cidade do Rio de Janeiro (RJ) do ano de 2005, com o objetivo de conhecer a magnitude e as características associadas à gravidez recorrente (GR). Foram estimadas razões de prevalência de GR com intervalos de confiança (IC) de 95\% para variáveis selecionadas através de regressão multivariada log-binomial. Entre 12.168 adolescentes, identificou-se prevalência de GR de 29,1\%. Os principais fatores associados à GR foram: idade 15-19 anos ( $R P=5,42$ IC95\% 3,72-7,81); não realizar pré-natal $(R P=2,36$ IC95\% 2,16-2,58); escolaridade $<4$ anos $(R P=1,48$ IC95\% 1,25-1,76); ocupação dona de casa $(R P=1,8$ IC 95\% 1,57-2,15) ou outra ( $R P=1,9$ IC95\% 1,732,10). Parto cesáreo e baixo peso foram associados negativamente a GR com RP iguais a 0,94 (IC95\% 0,86-0,99) e 0,69 (IC95\% 0,62-0,77). As adolescentes com gravidez recorrente tiveram indicadores socioeconômicos e assistenciais piores do que aquelas na primeira gravidez. Politicas sociais específicas para adolescentes mães em situação de vulnerabilidade possibilitariam melhores condições para o exercício de seus direitos reprodutivos. Palavras-chave Gravidez na adolescência, Vulnerabilidade social, Multiparidade 


\section{Introdução}

No Brasil, observou-se uma notável queda da fecundidade total e da fecundidade específica em praticamente todos os grupos etários, entre as décadas de 1970 e 2000, exceto no de 15 a 19 anos. Nesse grupo, a taxa de fecundidade cresceu $14 \%$, enquanto nas demais faixas etárias houve reduções de $43 \%$ a $93 \%{ }^{1}$. Nos anos recentes, as Pesquisas Nacionais por Amostra Domiciliar (PNAD) de 2002, 2004 e 2006 e do Sistema de Informação de Nascidos Vivos (Sinasc) indicam uma tendência de declínio das taxas de fecundidade de adolescentes ${ }^{2,3}$.

A questão da reprodução na adolescência tem sido tema de discussões e fonte de preocupações para autoridades públicas, profissionais de saúde e educação, pesquisadores de várias áreas disciplinares e a sociedade em geral, no Brasil e no mundo. Há vasta literatura dedicada a investigar o fenômeno e são numerosos os objetos sobre os quais se tem debruçado: correlações entre maternidade/paternidade e vulnerabilidades socioeconômicas; riscos obstétricos e perinatais; aborto inseguro; acesso a serviços de saúde sexual e reprodutiva; conhecimentos e utilização de métodos contraceptivos; questões socioantropológicas sobre sexualidade e reprodução adolescente, entre outros ${ }^{4-9}$.

Um objeto de estudo considerado pouco explorado no Brasil é a recorrência da gravidez no período da adolescência. Ao revisarem a produção científica sobre gravidez sucessiva na adolescência, Rosa et al. ${ }^{10}$ encontraram poucos trabalhos que investigam o fenômeno de modo mais consistente, e grande parte das vezes o assunto é apenas citado em estudos sobre a gestação na adolescência. Os autores concluíram que o tema ainda não se firmou no campo da saúde pública.

A recente Pesquisa Nacional Demografia e Saúde $2006^{11}$ revelou que $16,2 \%$ de mulheres na faixa etária de 15-19 anos já eram mães, e entre estas, $13,5 \%$ tinham dois filhos ou mais. Esses resultados confirmam a pertinência de se investir na produção de mais conhecimento sobre a magnitude, a distribuição e as características associadas à gravidez recorrente na adolescência, de modo a contribuir para o avanço do debate dos direitos reprodutivos dessa parcela da população e para o fortalecimento de políticas que visam assegurá-los.

O objetivo deste artigo é descrever o perfil de adolescentes residentes do município do Rio de Janeiro (RJ) que tiveram filhos nascidos vivos no ano de 2005 e identificar fatores associados à gra- videz recorrente nessa fase da vida, a partir dos dados do Sinasc. O estudo foi apoiado financeiramente e tecnicamente pela Organização PanAmericana da Saúde (Opas) e realizado em 2007 e 2008 .

\section{Materiais e métodos}

Foi realizado um estudo transversal utilizando informações das Declarações de Nascidos Vivos (DNV) de filhos de mães com idades entre $10 \mathrm{e}$ 19 anos residentes na cidade do Rio de Janeiro, referentes ao ano de 2005, obtidas no Sinasc, através do Datasus. A qualidade dos dados deste sistema tem sido considerada muito boa, e a maioria das variáveis apresenta um percentual de informação ignorada inferior a 3\%, embora os dados referentes a número de filhos tidos seja considerado regular ${ }^{12}$.

O desfecho deste estudo foi a gravidez recorrente na adolescência. Foi considerada gravidez recorrente quando no campo "número de filhos tidos em gestações anteriores" da DNV havia registro de pelo menos um filho tido anteriormente, vivo ou morto. Considerou-se como "primeira experiência de maternidade" quando a soma número de filhos tidos, vivos ou mortos, era igual a zero. As características sociodemográficas, de acesso aos serviços de saúde e obstétricas das adolescentes que tiveram mais de um filho vivo foram comparadas com aquelas das que tiveram a primeira experiência de maternidade.

Para identificar fatores associados, foram analisadas as seguintes variáveis: local de ocorrência do parto, idade, estado civil, escolaridade e ocupação habitual da mãe, duração da gestação, número de consultas de pré-natal, tipo de parto, tipo de estabelecimento, peso ao nascer, asfixia (Apgar no $5^{\circ}$ minuto $<8$ ) e raça/cor do bebê.

O perfil das adolescentes segundo o número de filhos vivos foi descrito através de percentuais. Também foram estimadas as associações entre as variáveis indicadas e a gravidez recorrente através do teste Qui-quadrado e das razões de prevalência com respectivos intervalos de confiança de 95\%, utilizando o programa SPSS15. As variáveis, cujas associações foram estatisticamente significativas $(\mathrm{p}<0,20)$ na análise univariada, foram selecionadas para a etapa da análise multivariada. Esta análise foi realizada através de regressão multivariada log-binomial, o que permitiu estimar a magnitude da associação de cada característica de forma independente. Nesta etapa, foram consideradas significativas as variáveis com $\mathrm{p}<0,05$. 


\section{Resultados}

No ano de 2005, do total de 84.228 nascidos vivos de mães residentes no município, $17,6 \%$ foram filhos de mães adolescentes, correspondendo a um número absoluto de 14.816 nascimentos. Foram excluídas da análise 2.648 DNV porque não havia preenchimento do campo "número de filhos tidos em gestações anteriores" ou por serem esses dados não compatíveis com a idade das adolescentes. Portanto, a população de estudo foi constituída por 12.168 indivíduos. Entre as 12.168 DNV analisadas, foram identificadas 8.626 adolescentes com primeira experiência de maternidade e 3.542 adolescentes com gravidez recorrente, correspondendo a $29,1 \%$ dos partos. Ao considerar a idade, nota-se que não havia casos de recorrência entre as adolescentes de 11 anos e apenas um caso entre as de 12 anos. Aos 13 anos, a frequência de gravidez recorrente entre as adolescentes que tiveram filhos foi de $3,2 \%$, ocorrendo um aumento gradativo deste percentual até que aos 19 anos corresponde a $42,5 \%$ daquelas que tiveram filhos em 2005 (Gráfico 1).

A Tabela 1 apresenta a distribuição proporcional das características epidemiológicas, socioeconômicas e de acesso aos serviços nos dois grupos. A comparação entre elas revela que as adolescentes com experiência de gravidez recorrente têm um perfil diferenciado daquelas que experimentaram a maternidade pela primeira vez, observando-se diferenças estatisticamente significativas para a maioria das variáveis estudadas com exceção da prematuridade.

Em relação à idade, observa-se que quase a totalidade das adolescentes com gravidez recorrente está na faixa etária de 15 a 19 anos (99,2\%). Apesar de o grupo da gravidez recorrente concentrar as adolescentes mais velhas, o nível de escolaridade observado foi baixo - somente $37,8 \%$ delas possuíam oito anos ou mais de estudos - e inferior àquele das meninas com uma única experiência de maternidade, entre as quais mais da metade atingia essa faixa. Há um predomínio de donas de casa na população estudada. Entre as adolescentes com mais de um filho, o percentual de estudantes é de somente $12,5 \%$, enquanto no outro grupo chega a aproximadamente $30 \%$. Mais de $90 \%$ das jovens eram solteiras nos dois grupos, com um percentual superior em $1 \%$ para aquelas com gravidez recorrente.

Quanto ao acompanhamento pré-natal, as gestantes com experiência anterior de maternidade realizaram um número menor de consultas - um quarto não chegou a realizar quatro con- sultas. Na assistência ao parto, há maior proporção de partos realizados no setor financiado pelo Sistema Único de Saúde (SUS) (90\%) neste grupo. Em relação ao tipo de parto, existe um menor percentual de cesarianas entre as jovens com gravidez recorrente, embora nos dois grupos a frequência deste procedimento seja considerada elevada ( $25 \%$ e $30 \%)$ (Tabela 1$)$.

O índice de prematuridade foi $8,9 \%$, sem diferença estatística entre os dois grupos, porém entre as adolescentes com mais de um filho, os percentuais de baixo peso $(9,5 \%)$ e de asfixia $(4,0 \%)$ foram menores. Havia uma proporção maior de filhos de cor parda ou preta no grupo com gravidez recorrente (63\%), conforme a Tabela 1.

A prevalência de gravidez recorrente foi de $29 \%$. Na análise da razão de prevalência ajustada, é possível identificar que idade, escolaridade, ocupação e menor número de consultas pré-natais foram fatores que permaneceram positivamente associados à gravidez recorrente. Jovens com mais de 15 anos apresentam uma prevalência 5,42 (IC95\% 3,72-7,81) vezes maior de gravidez recorrente quando comparadas com aquelas de 10-14 anos. Também se observou que quanto menor a escolaridade maior a proporção de gravidez recorrente: $41,95 \%$ das adolescentes com escolaridade entre 0-3 anos já tinham pelo menos dois filhos, o que representou uma frequência 1,48 vez maior em comparação com o grupo com mais de 11 anos de escolaridade (Tabela 2).

Além disso, chama a atenção o fato de que entre aquelas adolescentes que não fizeram pré-

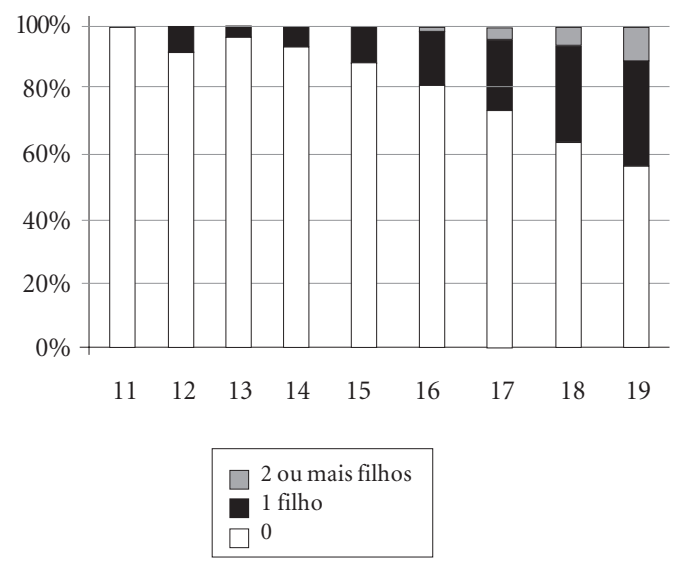

Gráfico 1. Número de filhos segundo a idade. 
natal, em que $2 / 3$ se referem ao grupo com gravidez recorrente, a prevalência de gravidez recorrente foi 2,36 (IC 95\% 2,16-2,58) vezes maior do que aquela observada entre as que tiveram sete consultas pré-natais ou mais. Quanto às variáveis indicadoras de maior risco para o bebê, ob- servou-se que o peso ao nascer se associou negativamente $(\mathrm{RP}=0,69$; IC95\% 0,62-0,77), porém não foram evidenciadas diferenças estatisticamente significativas entre prevalências de gravidez recorrente nas diferentes categorias de Apgar e de prematuridade.

Tabela 1. Distribuição proporcional das características de mães adolescentes - Município do Rio de Janeiro (RJ), 2005.

\begin{tabular}{|c|c|c|c|c|c|}
\hline & \multicolumn{2}{|c|}{$\begin{array}{l}1^{\text {a }} \text { experiência de } \\
\text { maternidade }\end{array}$} & \multicolumn{2}{|c|}{$\begin{array}{c}\text { Gravidez recorrente } \\
\qquad(\mathrm{n}=3.542)\end{array}$} & \multirow[t]{2}{*}{$\mathrm{p}$} \\
\hline & $\mathrm{N}$ & $\%$ & $\mathbf{N}$ & $\%$ & \\
\hline Idade da mãe $(\mathrm{n}=12.168)$ & & & & & $<0,001$ \\
\hline $10-14$ & 486 & 5,6 & 28 & 0,8 & \\
\hline $15-19$ & 8.140 & 94,4 & 3.514 & 99,2 & \\
\hline Estado civil $(\mathrm{n}=12.026)$ & & & & & 0,03 \\
\hline Solteira & 7.988 & 93,5 & 3.292 & 94,5 & \\
\hline Casadas/unidas & 551 & 6,4 & 189 & 5,4 & \\
\hline Viúvas/separadas & 5 & 0,1 & 1 & 0,1 & \\
\hline Escolaridade $(\mathrm{n}=11.898)$ & & & & & $<0,001$ \\
\hline Nenhuma & 21 & 0,2 & 34 & 1,0 & \\
\hline $1-3$ anos & 282 & 3,4 & 185 & 5,3 & \\
\hline 4-7 anos & 3.512 & 41,8 & 1.951 & 55,9 & \\
\hline $8-11$ anos & 3.880 & 46,1 & 1.127 & 32,3 & \\
\hline 12 anos ou mais & 714 & 8,5 & 192 & 5,5 & \\
\hline Ocupação habitual (n=12.012) & & & & & $<0,001$ \\
\hline Estudante & 2.575 & 30,2 & 435 & 12,5 & \\
\hline Dona de casa & 5.510 & 65,6 & 2.855 & 82,1 & \\
\hline Outra & 449 & 5,3 & 188 & 5,4 & \\
\hline Duração da gestação $(\mathrm{n}=12.076)$ & & & & & 0,22 \\
\hline Até 36 semanas & 822 & 9,6 & 312 & 8,9 & \\
\hline 37 semanas ou mais & 7.744 & 90,4 & 3.198 & 91,1 & \\
\hline Tipo de parto $(\mathrm{n}=12.134)$ & & & & & $<0,001$ \\
\hline Normal & 5.967 & 69,3 & 2.642 & 74,9 & \\
\hline Cesáreo & 2.638 & 30,7 & 887 & 25,1 & \\
\hline No de consultas pré-natal $(\mathrm{n}=11.897)$ & & & & & $<0,001$ \\
\hline Nenhuma consulta & 185 & 2,2 & 315 & 9,2 & \\
\hline $1-3$ consultas & 722 & 8,5 & 536 & 15,6 & \\
\hline $4-6$ consultas & 2.589 & 30,6 & 1.162 & 33,8 & \\
\hline 7 ou mais consultas & 4.961 & 58,7 & 1.427 & 41,5 & \\
\hline Peso ao nascer $(n=12.133)$ & & & & & $<0,001$ \\
\hline$<2.500$ gramas & 1.032 & 12,0 & 337 & 9,5 & \\
\hline 2.500 gramas ou mais & 7.568 & 88,0 & 3.196 & 90,5 & \\
\hline Raça/cor do bebê $(\mathrm{n}=11.946)$ & & & & & $<0,001$ \\
\hline Branca & 3.620 & 42,6 & 1.273 & 36,8 & \\
\hline Preta/parda & 4.854 & 57,2 & 2.174 & 63,0 & \\
\hline Outra & 18 & 0,2 & 7 & 0,2 & \\
\hline Apgar $(n=12.163)$ & & & & & 0,007 \\
\hline$<8$ & 442 & 5,1 & 139 & 4,0 & \\
\hline $8-10$ & 8.156 & 94,9 & 3.346 & 96,0 & \\
\hline Tipo de estabelecimento $(\mathrm{n}=11.564)$ & & & & & $<0,001$ \\
\hline SUS & 7.033 & 85,6 & 3.023 & 90,3 & \\
\hline Não SUS & 1.185 & 14,4 & 323 & 9,7 & \\
\hline
\end{tabular}


Tabela 2. Prevalência e razões de prevalências (RP) bruta e ajustada da gravidez recorrente para os fatores descritos na Declaração de Nascidos Vivos - Município do Rio de Janeiro (RJ), 2005.

\begin{tabular}{|c|c|c|c|c|c|}
\hline & \multirow{2}{*}{$\begin{array}{c}\text { Total de } \\
\text { adolescentes } \\
(\mathrm{n}=12.168)\end{array}$} & \multicolumn{2}{|c|}{$\begin{array}{l}\text { Gravidez recorrente } \\
\qquad(\mathrm{n}=3.542)\end{array}$} & \multirow{2}{*}{$\begin{array}{c}\text { Razão de } \\
\text { prevalência } \\
\text { bruta }\end{array}$} & \multirow{2}{*}{$\begin{array}{c}\text { Razão de } \\
\text { prevalência } \\
\text { ajustada }\end{array}$} \\
\hline & & $\mathbf{n}$ & Prevalência (\%) & & \\
\hline \multicolumn{6}{|l|}{ Idade da mãe $(n=12.168)$} \\
\hline $10-14$ & 514 & 28 & 5,45 & 1,00 & 1,00 \\
\hline $15-19$ & 11.654 & 3.514 & 30,15 & $5,54(3,86-7,94)$ & $5,42(3,72-7,81)$ \\
\hline \multicolumn{6}{|l|}{ Estado civil $(\mathrm{n}=12.020)$} \\
\hline Solteira & 11.280 & 3.292 & 29,18 & $1,14(1,01-1,38)$ & \\
\hline Casadas/unidas & 740 & 189 & 25,54 & 1,00 & \\
\hline \multicolumn{6}{|l|}{ Escolaridade $(\mathrm{n}=11.898)$} \\
\hline Nenhuma-3 anos & 522 & 219 & 41,95 & $1,98(1,69-2,33)$ & $1,48(1,25-1,76)$ \\
\hline 4-7 anos & 5.463 & 1.951 & 35,71 & $1,69(1,48-1,92)$ & $1,39(1,21-1,60)$ \\
\hline $8-11$ anos & 5.007 & 1.127 & 22,51 & $1,06(0,93-1,22)$ & $0,99(0,86-1,15)$ \\
\hline 12 anos ou mais & 906 & 192 & 21,19 & 1,00 & 1,00 \\
\hline \multicolumn{6}{|c|}{ Ocupação habitual $(\mathrm{n}=12.012)$} \\
\hline Estudante & 3.010 & 435 & 14,45 & 1,00 & 1,00 \\
\hline Dona de casa & 8.365 & 2.855 & 34,13 & $2,38(2,17-2,61)$ & $1,84(1,57-2,15)$ \\
\hline Outra & 637 & 188 & 29,51 & $2,04(1,76-2,37)$ & $1,91(1,73-2,10)$ \\
\hline \multicolumn{6}{|c|}{ Duração da gestação $(\mathrm{n}=12.076)$} \\
\hline Até 36 semanas & 1.134 & 312 & 27,51 & $0,94(0,85-1,04)$ & $0,92(0,86-0,99)$ \\
\hline 37 semanas ou mais & 10.942 & 3.198 & 29,23 & 1,00 & 1,00 \\
\hline \multicolumn{6}{|l|}{ Tipo de parto $(\mathrm{n}=12.134)$} \\
\hline Normal & 8.609 & 2.642 & 30,69 & $0,82(0,77-0,88)$ & $0,92(0,86-0,99)$ \\
\hline Cesáreo & 3.525 & 887 & 25,16 & 1,00 & 1,00 \\
\hline \multicolumn{6}{|c|}{ No de consultas pré-natal $(\mathrm{n}=11.897)$} \\
\hline Nenhuma consulta & 500 & 315 & 63,00 & $2,83(2,60-3,06)$ & $2,36(2,16-2,58)$ \\
\hline $1-3$ consultas & 1.258 & 536 & 42,61 & $1,91(1,76-2,06)$ & $1,81(1,66-1,98)$ \\
\hline 4-6 consultas & 3.751 & 1.162 & 30,98 & $1,39(1,30-1,48)$ & $1,36(1,26-1,46)$ \\
\hline 7 ou mais consultas & 6.388 & 1.427 & 22,34 & 1,00 & 1,00 \\
\hline \multicolumn{6}{|l|}{ Peso ao nascer $(n=12.133)$} \\
\hline$<2500$ gramas & 1.369 & 337 & 24,62 & $0,83(0,75-0,91)$ & $0,69(0,62-0,77)$ \\
\hline 2500 gramas ou mais & 10.764 & 3.196 & 29,69 & 1,00 & 1,00 \\
\hline \multicolumn{6}{|c|}{ Raça/cor do bebê $(\mathrm{n}=11.921)$} \\
\hline Branca & 4.893 & 1.273 & 26,02 & 1,00 & \\
\hline Preta/parda & 7.028 & 2.174 & 30,93 & $1,49(1,40-1,58)$ & \\
\hline \multicolumn{6}{|l|}{ Apgar $(n=12.163)$} \\
\hline$<8$ & 256 & 63 & 24,61 & $0,85(0,69-1,06)$ & \\
\hline $8-10$ & 11.827 & 3.422 & 28,93 & 1,00 & \\
\hline \multicolumn{6}{|c|}{ Tipo de estabelecimento $(n=11.564)$} \\
\hline SUS & 10.056 & 3.023 & 30,06 & $1,70(1,54-1,89)$ & \\
\hline Não SUS & 1.508 & 323 & 21,42 & 1,00 & \\
\hline
\end{tabular}

\section{Discussão}

A prevalência da gestação recorrente entre mulheres adolescentes residentes no município do Rio de Janeiro que tiveram filhos no ano de 2005 foi de $29,2 \%$, bastante superior àqueles percentuais encontrados em estudos realizados com outras amostras de mães adolescentes das regiões Sul e Sudeste, que variaram de $9,5 \%$ a $19,7 \%{ }^{13-}$
${ }^{15}$. Nesse sentido, os índices do município se aproximariam mais daqueles encontrados em estudos com populações do Norte e do Nordeste, nos quais foram registrados percentuais de recorrência entre $26,2 \%$ e $46,2 \%{ }^{5,16,17}$. A PNDS $2006^{11}$ revelou que $13,5 \%$ das adolescentes mães tinham dois filhos ou mais.

Nesta pesquisa, foi encontrada associação entre a recorrência da gravidez na adolescência e 
algumas variáveis específicas, como idade, escolaridade, ocupação, tipo de parto, número de consultas pré-natal e peso ao nascer. A comparação entre adolescentes primíparas e multíparas indica um quadro de maior vulnerabilidade social entre as segundas, que têm menos acesso a educação, serviços de saúde e mercado de trabalho - evidenciado pela menor escolaridade, maior abandono dos estudos, maior proporção de jovens que, além de estarem fora da escola, tampouco estão no mercado de trabalho $(82,1 \%$ se declararam donas de casa), e menor frequência ao pré-natal. Esses achados são condizentes com a literatura. Variáveis relacionadas com o desempenho escolar (baixo nível de escolaridade, repetência, interrupções e abandono dos estudos) têm sido fortemente associadas à ocorrência de gravidez adolescente, gestações sucessivas e gestações de rápida repetição nesse período da vida ${ }^{4-}$ 6,10,15,18-22. Quanto ao pré-natal, o estudo mostra que as gestantes com experiência anterior de maternidade haviam realizado um número menor de consultas, sendo que $9 \%$ das multíparas e $2,2 \%$ das primíparas não haviam realizado nenhuma visita pré-natal. Um estudo com 1.967 puérperas adolescentes no município do Rio de Janeiro também observou que o número de consultas pré-natal diminuía à medida que crescia o número de nascidos vivos anteriores ${ }^{23}$. Por sua vez, outro estudo que comparou características demográficas, obstétricas e psicossociais de 616 adolescentes paulistas, primigestas e multigestas concluiu que não houve diferenças estatisticamente significativas entre esses grupos em número de consultas de pré-natal ${ }^{24}$. Os percentuais de adolescentes que não fizeram pré-natal encontrado na presente pesquisa chamam a atenção por estarem bem acima daqueles encontrados na PNDS 2006, que mostrou que apenas 1,3\% das gestantes adolescentes brasileiras não haviam realizado pré-natal.

Em relação ao tipo de parto, o estudo mostrou que o parto vaginal foi mais frequente entre as multíparas $(75 \%)$ do que entre primíparas (69\%). Achados similares foram os de Maia et al. ${ }^{16}$, que, analisando as vias de parto em 714 adolescentes, observaram cerca de $61 \%$ de partos vaginais em primíparas e de $65 \%$ entre aquelas que estavam parindo o segundo ou mais filhos.

Neste estudo, encontrou-se uma proporção de casadas e unidas no grupo das adolescentes com gestação recorrente $(5,4 \%)$ bem inferior àquelas encontradas em outros estudos, cujos percentuais de meninas com mais de um filho unidas variavam entre $67 \%$ e $75 \%^{10,18,24}$.
No que diz respeito às condições de saúde do bebê, os achados da pesquisa divergem do estudo de Waissman ${ }^{24}$, que observou maior probabilidade de uma adolescente multigesta ter um bebê prematuro do que uma primigesta. Em relação ao peso dos recém-nascidos, os estudos revisados divergem. McCormick et al. ${ }^{25}$, em estudo realizado nos Estados Unidos, encontraram maior percentual de recém-nascidos de baixo peso entre adolescentes multíparas. Outros estudos ${ }^{24,26}$ não encontraram diferenças estatisticamente significantes entre esses dois grupos. Diferente desses outros, o presente estudo encontrou uma proporção maior de baixo peso entre as primíparas, com significância estatística, convergindo com os resultados de pesquisa americana de Blankson et $a l .{ }^{27}$, que encontraram um peso médio ao nascer significativamente maior no segundo nascimento. A respeito dessa questão, Klerman ${ }^{28}$ discute a ideia de que, apesar de algumas pesquisas mostrarem que o segundo nascimento em adolescente geralmente tem resultados piores em termos de desfechos perinatais, os estudos longitudinais mostram que individualmente as adolescentes não têm maior risco para baixo peso e os problemas a ele associados numa segunda gestação. Os achados dos estudos longitudinais são semelhantes aos observados nas mulheres adultas, em que a segunda gravidez traz menos riscos para o bebê que a primeira gravidez.

Como limite inerente ao estudo, podemos apontar o fato de a nossa fonte de informação fornecer dados sobre o número de filhos tidos em gestações anteriores, e não sobre o número de gestações. Por esta razão, a história de gravidez terminada em aborto não está sendo captada, e não podemos afirmar se, de fato, a totalidade dessas primíparas está em curso da primeira gravidez. Esse limite não compromete os resultados encontrados, pois o tipo de distorção gerado seria provavelmente devido à inclusão, no grupo de primíparas, de gestantes que já poderiam estar na segunda gravidez ou mesmo ainda vir a ter um segundo filho. Como se sabe, este grupo se caracteriza por apresentar menor escolaridade e menor acesso ao serviço de saúde. Caso tenha ocorrido algum viés, a análise sugere que seja na direção de uma redução do nível de significância ou mesmo da magnitude das associações observadas.

Não foi possível dispor da informação sobre número de filhos de 2.648 adolescentes. Este grupo apresentava maior escolaridade, maior número de consultas pré-natais e maior percentual de bebês de cor/raça branca em comparação com 
a população analisada neste estudo, o que sugere uma proporção maior de primíparas neste grupo. Portanto, as diferenças entre o perfil das adolescentes na primeira gravidez e aquelas de gravidez recorrente devem ser ainda maiores.

A comparação dos resultados do presente estudo com outros realizados no país - tanto no que diz respeito à magnitude do fenômeno da gravidez recorrente como na sua associação com fatores socioeconômicos, demográficos e assistenciais - deve ser cautelosa, pois os estudos têm metodologias, abrangência e recortes temporais bastante diferenciados.

\section{Considerações finais}

Estudos multidisciplinares têm apontado a tendência de uma parcela significativa de adolescentes e jovens de constituir prole nesse período de suas vidas, muitos de modo pró-ativo e/ou assertivo e em contexto de relacionamentos estáveis ${ }^{4,29}$. Por sua vez, condizente com os resultados da pesquisa aqui apresentada, vários desses estudos demonstram que muitos adolescentes e jovens vivenciam a maternidade/paternidade em condições de forte iniquidade social, com comprometimento de sua qualidade de vida e de seus direitos, assim como os de suas famílias. A "gravidez recorrente" parece representar emblematicamente essa realidade, pois essa população apresenta indicadores sociais ainda piores. Isso remete à reflexão sobre a necessidade de se deslocar o foco central de análise do marcador biológico da "fecundidade/gravidez" para "vivência da maternidade/paternidade de jovens e adolescentes e seus contextos". A ampliação do olhar sobre o tema em questão tem implicações relevantes tanto para o campo do conhecimento como para o campo das políticas públicas.
A promoção e a prevenção em saúde sexual e reprodutiva dos adolescentes e jovens têm sido um dos eixos prioritários das políticas voltadas para os adolescentes ${ }^{30,31}$. No histórico de implementação dessas políticas, um enfoque por vezes excessivamente centrado na prevenção da gravidez "não desejada", "não planejada" ou "precoce" tem obscurecido tanto aspectos mais abrangentes do direito à atenção integral à saúde sexual e reprodutiva como outras obrigações do Estado e da sociedade em relação aos direitos dessa população.

A Constituição Federal de 1988 elenca no rol dos direitos sociais - que são parte dos direitos e garantias fundamentais de todo cidadão e cidadã brasileira - a proteção social à maternidade. $\mathrm{Na}$ perspectiva ampliada sugerida aqui, o desafio diante do fenômeno da reprodução adolescente não se restringe ao problema de como prevenir gravidezes consideradas "inoportunas" - consideração que muitas vezes expressa a visão de agentes externos, e não necessariamente dos próprios adolescentes - mas sim como promover o direito fundamental à proteção social da maternidade. Políticas intersetoriais e integradas especificas para mães e pais adolescentes e jovens em situação de vulnerabilidade social, na perspectiva do trabalho em "rede de proteção" 32 - com programas de renda, creches, reinserção no sistema escolar, qualificação profissional e estratégias para maior utilização dos serviços de promoção à saúde sexual e reprodutiva -, poderiam ser instrumentos inestimáveis para habilitar essas pessoas para o exercício dos direitos reprodutivos. A perpetuação da situação de falta de poder, reconhecimento e precariedade de acesso a recursos sociais após a experiência da maternidade, em círculo vicioso, compromete a capacidade desses sujeitos de controlar vários aspectos de sua vida, inclusive tomar decisões sobre o número e o espaçamento entre filhos e efetivá-las.

\section{Colaboradores}

KS Silva trabalhou na análise de dados para a elaboração do artigo; R Rozenberg, VCC Chuva e SF Costa participaram do projeto inicial; C Bonan e MASM Gomes trabalharam na elaboração do texto e na revisão final. 


\section{Referências}

1. Brasil. Ministério da Saúde. Secretaria de Vigilância em Saúde. Saúde - Brasil 2004: uma análise da situação de saúde. Brasília: Ministério da Saúde; 2004.

2. Berquó E, Cavenaghi S. Increasing adolescent and youth fertility in Brazil: a new trend or a one-time wvent? In: Annals Population Association of America. Annual Meeting, Session 151. Pennsylvania, Philadelphia; 2005. p. 18.

3. Barbosa AM. Análise sociodemográfica da fecundidade de adolescentes e jovens no Brasil: 1970/ 2006. In: XVI Encontro Nacional de Estudos Populacionais; 2008 set 29-out 3; Caxambu, MG, Brasil.[documento na Internet] [acessado 2009 jan 20]. Disponível: http://www.abep.nepo.unicamp.br/ encontro2008/docspdf/ABEP2008_1206.pdf

4. Heilborn ML, Aquino EML, Bozon M, Knauth DR, organizadores. O aprendizado da sexualidade: reprodução e trajetórias sociais de jovens brasileiros. Rio de Janeiro: Editora Garamond/Editora Fiocruz; 2006.

5. Simões VMF, Silva AMM, Bettiol H, Lamy-Filho F, Tonial SR, Mochel EG. Características da gravidez na adolescência em São Luís do Maranhão. Rev Saude Publica 2003; 3:559-565.

6. Duarte CM, Nascimento VB, Akerman M. Gravidez na adolescência e exclusão social: análise de disparidades intra-urbanas. Rev Panam Salud Publica/Pan Am J Public Health 2006; 19(4):236-243.

7. Silva L, Tonete VLP. A gravidez na adolescência sob a perspectiva dos familiares: compartilhando projetos de vida e cuidado. Rev Lat Am Enfermagem 2006; 14(2):199-206.

8. Vieira LM, Goldberg TBL, Saes SO, Doria AAB. Abortamento na adolescência: um estudo epidemiológico. Cien Saude Colet 2007; 12(5):1201-1208.

9. Alves CA, Brandão ER. Vulnerabilidades no uso de métodos contraceptivos entre adolescentes e jovens: interseções entre políticas públicas e atenção à saúde. Cien Saude Colet 2009; 14(2):661-670.

10. Rosa AJ, Reis AOA, Tanaka ACd'A. Gestações sucessivas na adolescência. Rev Bras Crescimento Desenvolv Hum 2007; 17:165-172.

11. Brasil. Ministério da Saúde. PNDS 2006 - Pesquisa Nacional de Demografia e Saúde da Criança e da Mulher: relatório final. Brasília: Ministério da Saúde; 2008. [documento na Internet] 2008. [acessado 2009 jan 3]. Disponível em: http://bvsms.saude.gov.br/ bvs/pnds/img/relatorio_final_pnds2006.pdf

12. Romero DE, Cunha CB. Sistema de Informações sobre Nascidos Vivos, 2002. Cad Saude Publica 2007; 23(3):701-714.

13. Siqueira MJT, Mendes D, Finkler I, Guedes T, Gonçalves MDS. Profissionais e usuárias(os) adolescentes de quatro programas públicos de atendimento pré-natal da região da Grande Florianópolis: onde está o pai? Estud Psicol 2002; 7(1):65-72.
14. Rugolo LMSS, Bottino J, Scudeler SEM, Bentlin MR, Trindade CP, Perosa B, Rugolo Júnior A. Sentimentos e percepções de puérperas com relação à assistência prestada pelo serviço materno-infantil de um hospital universitário. Rev Bras Saúde Matern Infant 2004; 4(4):423-433.

15. Yazaki LM. Maternidades sucessivas em adolescentes no Estado de São Paulo. In: XVI Encontro Nacional de Estudos Populacionais, realizado em Caxambu MG - Brasil; 2008 set 29-out 3; Caxambu; 2008. [acessado 2009 jan 15]. Disponível em: http:// www.abep.nepo.unicamp.br/encontro2008/docs PDF/ABEP2008_1170.pdf

16. Maia VAO, Maia ACA, Queiroga FL, Filho VOAM, Araújo AB, Lippo LAM, Albuquerque RM. Via de parto em gestações sucessivas em adolescentes: estudo de 714 casos. Ver Bras Ginecol Obstet 2004; 26:703-707.

17. Chaban JRN, Maquera ERA, Lopes CM. Prevalência da gravidez em adolescentes em uma vila, AcreBrasil. Online Brazilian Journal of Nursing [periódico na Internet]. 2003 [acessado 2009 jan 15]; 2(3): [cerca de 12p.]. Disponível em: http://www.uff.br/ nepae/objn203chabanjunioetal.htm

18. Persona L. Perfil de adolescentes com repetição da gravidez. Rev Latino Am Enfermagem 2004; 12:745750.

19. Castro MG, Abramovay M, Silva LB. Juventudes e sexualidade. Brasília: Unesco; 2004.

20. Gomes SEC. Gravidez na adolescência e sua recorrência [dissertação]. São Paulo: Escola de Enfermagem, Universidade de São Paulo; 2004.

21. Magalhães RR. A gravidez recorrente na adolescência: o caso de uma maternidade [tese]. Rio de Janeiro: Pós-Graduação em Saúde da Criança e da Mulher, Instituto Fernandes Figueira, Fundação Oswaldo Cruz; 2001.

22. Sant'Anna MJ, Carvalho KA, Melhado A, Coates V, Omar HA. Teenage pregnancy: impact of the integral attention given to the pregnant teenager and adolescent mother as a protective factor for repeat pregnancy. Scientific World Journal 2007; 7:187-194.

23. Gama SGN, Szwarcwald CL, Sabroza AR, Castelo Branco V, Leal MC. Fatores associados à assistência pré-natal precária em uma amostra de puérperas adolescentes em maternidades do município do Rio de Janeiro, 1999-2000. Cad Saude Publica 2004; 20(Supl.1):S101-S111.

24. Waissman AL. Análise dos fatores associados à recorrência da gravidez na adolescência [tese]. São Paulo: Faculdade de Medicina da Universidade de São Paulo; 2006. [acessado 2009 jan 18]. Disponível em: http://www.teses.usp.br 
25. McCormick MC, Shapiro S, Starfield B. High-risk young mothers: infant mortality and morbidity in four areas in the United States, 1973-1978. Am J Public Health 1984; 74:18-23.

26. Santelli JS, Jacobson MS. Birth weight outcomes for repeat teenage pregnancy. J Adolesc Health Care 1990; 11:240-247.

27. Blankson ML, Cliver SP, Goldenberg RL, Hickey CA, Jin J, Dubard MB. Health behavior and outcomes in sequential pregnancies of black and white adolescents. JAMA 1993; 269:1401-1403.

28. Klerman LV. Risk of poor pregnancy outcomes: is it higher among multiparous teenage mothers? Journal of Adolescent Health 2006; 38:761-764.

29. Catharino TR. Da gestão dos riscos à intervenção do futuro: considerações médico-psicológicas e educacionais sobre meninas que engravidaram entre 10 e 14 anos [tese]. São Paulo: Instituto de Psicologia da Universidade de São Paulo; 2002.

30. Brasil. Ministério da Saúde. Departamento de Ações Programáticas Estratégicas. Diretrizes nacionais para a atenção integral à saúde de adolescentes e de jovens na promoção, proteção e recuperação da saúde. Brasília: Ministério da Saúde; 2010.

31. Brasil. Ministério da Saúde. Secretaria de Atenção à Saúde. Departamento de Ações Programáticas e Estratégicas. Marco teórico e referencial: saúde sexual e saúde reprodutiva de adolescentes e jovens. Brasília: Ministério da Saúde; 2006. (Série B. Textos Básicos de Saúde).

32. Costa MCO, Bigras M. Mecanismos pessoais e coletivos de proteção e promoção da qualidade de vida para a infância e adolescência. Cien Saude Colet 2007; 12(5):1101-1109.

Artigo apresentado em 05/05/2009

Aprovado em 05/10/2009

Versão final apresentada em 12/11/2009 